\title{
EFICIÊNCIA DE UM MODELO DE AFILAMENTO AJUSTADO SEM E COM ESTRATIFICAÇÃO POR CLASSE DE QUOCIENTE DE FORMA PARA FORMAÇÃO DOS SORTIMENTOS DE Pinus taeda L.
}

\author{
EFFICIENCY OF A TAPER MODEL ADJUSTED WITH AND WITHOUT TRATIFICATION BY \\ CLASS OF FORM QUOTIENT FOR THE ASSORTMENT OF Pinus taeda L.
}

Carlos Alberto Martinelli de Souza ${ }^{1}$ César Augusto Guimarães Finger ${ }^{2}$

Paulo Renato Schneider ${ }^{3}$ Gilson Fernandes da Silva ${ }^{4}$ Claúdio Thomas ${ }^{5}$

\begin{abstract}
RESUMO
Os objetivos deste trabalho foram modelar a forma de tronco de Pinus taeda L., comparar a eficiência na estimativa do volume total do polinômio do quinto grau ajustado com dados não estratificados e estratificados segundo quocientes de forma e apresentar os resultados em forma de tabela de sortimentos. As árvores amostradas foram medidas em povoamentos manejados para serraria, com dois desbastes, sendo o plantio inicial de 1.600 árvores/ha, em espaçamento de 2,50 x 2,50 m, localizados na Klabin S.A., em Telêmaco Borba-PR. Para fins de modelagem os dados foram estratificados em classes de quociente de forma calculado a $70 \%$ da altura. A classificação das partes do tronco em sortimentos foi acurada sendo que o volume estimado a partir das integrais das equações de afilamento apresentou valores residuais pequenos e sem tendências, comprovando a viabilidade da estratificação dos dados em classes de quociente de forma quando do ajuste de equações para a obtenção dos sortimentos.
\end{abstract}

Palavras-chave: manejo florestal; volume individual; classificação de toras.

\begin{abstract}
The objective of this paper was model the shape, compare the efficiency of the total volume estimated with the 5th grade polynomial, with and without data stratification by an artificial form quotient and build the assortment table. The data came from Pinus taeda L. stands, managed by a sawmill company, with two thinning and coppicing, plantation of 1.600 trees/ha and spacing of 2,50 x 2,50 m, from Klabin S.A., in Telêmaco Borba, Paraná state. The field data was stratified in three form quotient classes defined by the diameter of $70 \%$ total height and breast height diameter. The trunk classification in assortments was accurate and the volume obtained with the integrated equations presented small residues, without tendency. The results proved the viability of the data stratification by form quotients to obtain better estimative of tree assortments.
\end{abstract}

Keywords: forest management; individual volume; classification of logs.

1. Engenheiro Florestal, Dr., Professor Adjunto do Departamento de Engenharia Florestal, Centro de Ciências Agrárias, Universidade Federal do Espírito Santo, Campus de Alegre, CEP 29500-000, Alegre (ES). In memorium

2. Engenheiro Florestal, Dr., Professor Associado do Departamento de Ciências Florestais, Centro de Ciências Rurais, Universidade Federal de Santa Maria, Campus Universitário, CEP 97105-900, Santa Maria (RS). caesar.finger@gmail.com

3. Engenheiro Florestal, Dr., Professor Titular do Departamento de Ciências Florestais, Centro de Ciências Rurais, Universidade Federal de Santa Maria, Campus Universitário, CEP 97105-900, Santa Maria (RS). paulors@smail.ufsm.br

4. Engenheiro Florestal, Dr., Professor Adjunto do Departamento de Engenharia Florestal, Centro de Ciências Agrárias, Universidade Federal do Espírito Santo, Campus de Alegre, CEP 29500-000, Alegre (ES). gfsilva2000@yahoo.com

5. Engenheiro Florestal, M.Sc., Doutorando do Programa de Pós-graduação em Engenharia Florestal, Centro de Ciências Rurais, Universidade Federal de Santa Maria, Campus Universitário, CEP 97105-900, Santa Maria (RS). thomas.claudio@hotmail.com

Recebido para publicação em 13/04/2010 e aceito em 15/12/2010 


\section{INTRODUÇÃO}

No Brasil, a maior parte dos produtos advindos de florestas é originada de plantios com espécies dos gêneros Pinus e Eucalyptus, o que, dentre outras, diminui a pressão sobre as florestas naturais, principalmente as da Amazônia. Estas florestas, normalmente, estão vinculadas a empreendimentos empresariais, nos quais a tônica do negócio e a agregação de renda são cada vez maiores (SOUZA et al., 2008a).

As empresas produtoras de matériaprima florestal possuem, como um dos principais objetivos, a obtenção de retorno econômico a partir dos plantios que realizam. Esse retorno econômico está, entretanto, condicionado às limitações impostas pela qualidade da matéria-prima, preços, mercados, localização geográfica e transporte, dentre diversos outros fatores.

Uma característica comum do plantio florestal é que a demanda, geralmente, se concentra em um determinado tipo de produto para abastecer uma indústria, como madeira para processo (elaboração de pasta celulósica e papel) ou energia. Porém, em geral, as florestas produzem uma variedade maior de produtos como para laminação, serraria, , etc para os quais, nem sempre existe uma demanda localizada e próxima para outorgar algum tipo de valor a estes produtos (ARCE et al., 2004).

Por outro lado os desperdícios causados pelos atuais processos de transformação têm induzido à pesquisa e ao desenvolvimento de modelos estatísticos aplicados ao manejo de florestas que auxiliem na definição de uso dessas madeiras e de transformação em produto final, com o intuito de torná-las mais rentáveis (CHICHORRO et al., 2000)

Além disso, segundo Schneider et al. (1996), uma das grandes dificuldades do manejo florestal e, em especial, da avaliação econômica de povoamentos florestais, reside na inexistência de tabelas de sortimento apropriadas que possibilitem determinações rápidas do estoque de madeira para diferentes tipos de aproveitamento. Devido a isto, muitas pesquisas têm sido realizadas com o objetivo de descrever, de forma otimizada, a classificação dos fustes segundo sua qualidade, dimensões e possibilidades de utilização, garantindo, além da classificação física, uma melhor remuneração da madeira.

Portanto, as funções de forma ou de afilamento são uma excelente opção para quantificação dos sortimentos dos povoamentos florestais. A gama de informações que propiciam tem levado ao desenvolvimento de diferentes técnicas de modelagem do perfil dos fustes das espécies florestais. Mesmo com a quantidade de estudos referentes a este assunto já realizados, sempre existe a possibilidade de melhorar a eficiência de tais funções (SOUZA et al., 2008b).

Diante do exposto, os objetivos deste trabalho foram modelar a forma de tronco de Pinus taeda L., comparar a eficiência na estimativa do volume total com o polinômio do quinto grau ajustado com dados não estratificados e estratificados com base em quocientes de forma e, apresentar os sortimentos em forma de tabela.

\section{MATERIAL E MÉTODOS}

\section{Localização e descrição da área de coleta dos dados}

Os dados para o presente trabalho foram medidos em povoamentos de Pinus taeda L., localizados na Klabin S.A., em Telêmaco Borba$\mathrm{PR}$, a $24^{\circ} 08^{\prime}$ latitude sul e $50^{\circ} 30^{\prime}$ longitude oeste, com altitude de 750 a $868 \mathrm{~m}$.

Segundo classificação de Köppen, o clima local é Cfa, subtropical, apresentando temperatura média no mês mais frio de $15,6^{\circ} \mathrm{C}$, temperatura média no mês mais quente $22,3^{\circ} \mathrm{C}$ e temperatura mínima registrada de $-5,2^{\circ} \mathrm{C}$. A precipitação média anual nos últimos 54 anos é de 1.508,8 mm.

Os solos predominantes na região são o Latossolo e o Cambissolo, com textura argilosa e média. O material de origem é, predominantemente, relacionado ao intemperismo e retrabalhamento de litologias referentes às formações Rio Bonito, Itararé e Diques de Diabásio (EMBRAPA, 2006).

\section{Características do povoamento}

As árvores amostradas cresceram em povoamentos manejados para serraria, com dois desbastes e corte raso aos 30 anos. O plantio foi realizado com 1.600 árvores/ha em espaçamento de $2,50 \times 2,50 \mathrm{~m}$. O primeiro desbaste, realizado aos nove anos, foi sistemático na sexta linha e seletivo entre estas, permanecendo 675 árvores/ha. O segundo desbaste, do tipo seletivo, foi realizado aos 14 anos permanecendo 275 árvores/ha.

\section{Informações coletadas}

Quarenta árvores foram abatidas e cubadas

Ci. Fl., v. 22, n. 1, jan.-mar., 2012 
pelo Método de Smalian nas posições de: 0,10; 0,$30 ; 0,80 ; 1,30$ e, a partir desta posição, de metro em metro até a altura total. Nestas posições foram retirados discos transversais de madeira para fins de estudo dendrocronológico segundo a metodologia apresentada por Finger (1992). Com a reconstrução do crescimento e das dimensões das árvores em diferentes idades foram obtidos perfis de 649 árvores. Destes, noventa e seis, com idades variando de 8 a 30 anos, distribuídos conforme apresentado na Tabela 1, foram selecionados para a modelagem.

\section{Métodos de estratificação dos dados para estimativa dos volumes}

O polinômio do quinto grau foi ajustado separadamente a dados não estratificados e estratificados em classes de quociente de forma $\left(\mathrm{K}_{0,7 \mathrm{~h}}\right)$. A opção por $\mathrm{K}_{0,7 \mathrm{~h}}$ foi baseada em trabalho publicado por Souza (2009) que comparou a acurácia de modelos de sortimento quando os dados foram classificados em classes de $\mathrm{K}_{0,7 \mathrm{~h}}, \mathrm{~K}_{0,5 \mathrm{~h}}$ e $\mathrm{K}_{0,3 \mathrm{~h}}$.

Assim, os dados das árvores-amostra da Tabela 1 foram agrupados em três classes segundo os valores de $\mathrm{K}_{0,7 \mathrm{~h}}$, com centros em 0,$4 ; 0,5$ e 0,6 obtidos a partir da razão entre o diâmetro tomado a $70 \%$ da altura total da árvore $\left(\mathrm{d}_{0,7 \mathrm{~h}}\right)$ e o dap.

Desse modo quando uma árvore era classificada na classe 0,4 , a estimativa dos coeficientes do polinômio e, posteriormente, seu volume foi calculado com a equação de forma desta classe e, assim sucessivamente.

\section{Avaliação dos métodos testados}

Os resultados foram avaliados em relação à acurácia para a estimativa do volume total utilizando as estatísticas viés, média das diferenças absolutas $(m d)$ e desvio padrão das diferenças $(d p d)$, como descrito abaixo:

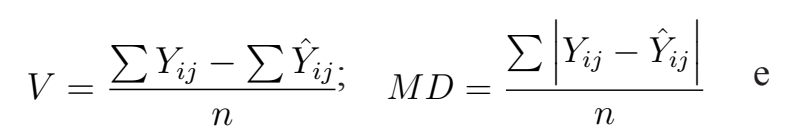

$$
\mathrm{D} P D=\sqrt{\left(\sum_{i=1}^{n} d_{i}{ }^{2}-\left(\sum_{i=1}^{n} d_{i}\right)^{2} / n\right) / n-p}
$$

Em que: $\mathrm{Yi}=$ valor observado e $\hat{\mathrm{Y}} \mathrm{i}=$ valor estimado; $n=$ número de observações; $\mathrm{e} p=$ número de parâmetros de cada modelo e $d_{i}=\left(Y_{i j}-\hat{Y}_{i j}\right)$.

Acada uma das estatísticas testadas atribuiuse nota um para o método com melhor acurácia e nota dois para o segundo e, assim sucessivamente até classificar o menos acurado.

$\mathrm{O}$ melhor método de estratificação dos dados ficou estabelecido como àquele que apresentou o menor valor no somatório das notas. O coeficiente de determinação e o coeficiente de variação foram utilizados ainda para determinar a precisão das equações, bem como a análise gráfica dos resíduos foi empregada na interpretação dos resultados.

TABELA 1: Distribuição de frequência das árvores-amostra por diâmetro e altura.

\begin{tabular}{|c|c|c|c|c|c|c|c|c|}
\hline \multirow{2}{*}{ Altura (m) } & \multicolumn{7}{|c|}{ Classes de diâmetro $(\mathrm{cm})$} & \multirow[b]{2}{*}{ Total } \\
\hline & 12,5 & 17,5 & 22,5 & 27,5 & 32,5 & 37,5 & 42,5 & \\
\hline 12 & 1 & 2 & & & & & & 3 \\
\hline 14 & 1 & 9 & 1 & & & & & 11 \\
\hline 16 & 2 & 7 & 5 & 1 & & & & 15 \\
\hline 18 & 1 & 4 & 2 & 2 & & & & 9 \\
\hline 20 & & 5 & 4 & 4 & & & & 13 \\
\hline 22 & & & 2 & 3 & 4 & & & 9 \\
\hline 24 & & & 1 & 5 & 3 & 2 & & 11 \\
\hline 26 & & & & 2 & 2 & 2 & & 6 \\
\hline 28 & & & & & 2 & 3 & & 5 \\
\hline 30 & & & & & 3 & 2 & & 5 \\
\hline 32 & & & & & 1 & 3 & 1 & 5 \\
\hline 34 & & & & & & & 3 & 3 \\
\hline 36 & & & & & & & 1 & 1 \\
\hline Total & 5 & 27 & 15 & 17 & 15 & 12 & 5 & 96 \\
\hline
\end{tabular}

TABLE 1: Frequency distribution of sample trees by diameter and height. 


\section{Sortimentos de madeira}

A integração de uma função de forma forneceu as estimativas de volume para todo o tronco e partes do mesmo, segundo limites diamétricos de utilização e comprimento de toras.

O procedimento para obtenção dos sortimentos partiu da integração do polinômio do quinto grau ajustado com os dados estratificados em classes de quocientes de forma com diâmetro obtido a 70\% da altura da árvore, tendo a equação expressa por:

$$
\begin{aligned}
\frac{d_{i}}{d}=b_{0}+ & b_{1} \cdot\left(\frac{h_{i}}{h}\right)+b_{2} \cdot\left(\frac{h_{i}}{h}\right)^{2}+b_{3} \cdot\left(\frac{h_{i}}{h}\right)^{3} \\
& +b_{4} \cdot\left(\frac{h_{i}}{h}\right)^{4}+b_{5} \cdot\left(\frac{h_{i}}{h}\right)^{5}
\end{aligned}
$$

Isolando $\mathrm{d}_{\mathrm{i}}$ na equação:

$$
\begin{aligned}
d_{i}=b_{0} \cdot d+ & \frac{\left(b_{1} \cdot d \cdot h_{i}\right)}{h}+\frac{\left(b_{2} \cdot d \cdot h_{i}^{2}\right)}{h^{2}}+\frac{\left(b_{3} \cdot d \cdot h_{i}^{3}\right)}{h^{3}} \\
+ & \frac{\left(b_{4} \cdot d \cdot h_{i}^{4}\right)}{h^{4}}+\frac{\left(b_{5} \cdot d \cdot h_{i}^{5}\right)}{h^{5}}
\end{aligned}
$$

Definindo os novos parâmetros como: $\mathrm{a}_{0}=$ $\mathrm{b}_{0} \cdot \mathrm{d} ; \mathrm{a}_{1}=\mathrm{b}_{1} \cdot(\mathrm{d} / \mathrm{h}) ; \mathrm{a}_{2}=\mathrm{b}_{2} \cdot\left(\mathrm{d} / \mathrm{h}^{2}\right) ; \mathrm{a}_{3}=\mathrm{b}_{3} \cdot\left(\mathrm{d} / \mathrm{h}^{3}\right) ; \mathrm{a}_{4}=$ $b_{4} \cdot\left(d / h^{4}\right) ; a_{5}=b_{5} \cdot\left(d / h^{5}\right)$, a equação de forma passou a ser escrita $d_{i}=a_{0}+a_{1} \cdot h_{i}+a_{2} \cdot h_{i}^{2}+a_{3} \cdot h_{i}^{3}+a_{4} \cdot h_{i}^{4}+$ $a_{5} \cdot h_{i}^{5}$

Integrando-se a equação acima entre zero (0) e a altura relativa $\left(\mathrm{h}_{\mathrm{i}}\right)$, obteve-se o volume absoluto da seguinte forma:

$$
v=K \cdot \int_{0}^{h_{i}} Y^{2} \cdot d_{h_{i}}
$$

Sendo: $K=(\pi / 4) / 10000=$ constante; $Y=d_{i}$ $=$ equação de forma de tronco redefinida; $h_{i}=$ altura relativa, correspondente ao comprimento da tora; $\mathrm{v}$ = volume, em metros cúbicos.

E, com isso, substituindo-se na equação a variável Y pela equação de di, obteve-se:

$$
\begin{gathered}
v=K \cdot \int_{0}^{h_{i}}\left(a_{0}+a_{1} \cdot h_{i}+a_{2} \cdot h_{i}^{2}+a_{3} \cdot h_{i}^{3}\right. \\
\left.+a_{4} \cdot h_{i}^{4}+a_{5} \cdot h_{i}^{5}\right)^{2} \cdot d_{h_{i}}
\end{gathered}
$$

Para o cálculo dos volumes dos sortimentos, utilizou-se a integração parcial da função de forma, desde a altura do toco até a altura na ponta mais fina da tora. O volume desses sortimentos resultou da subtração do volume obtido até o final da seção com o volume obtido até o início da seção, resultando na equação:

$$
v_{t}=K \cdot\left(\int_{0}^{X_{2}} Y^{2} \cdot d_{x_{2}}-\int_{0}^{X_{1}} Y^{2} \cdot d_{x_{1}}\right)
$$

Sendo que: $\mathrm{X}_{1}=$ altura no início da tora, em metros; $\mathrm{X}_{2}=$ altura no fim da tora, em metros; $\mathrm{v}_{\mathrm{t}}=$ volume da tora, em metros cúbicos; $K=(\pi / 4) / 10000$ $=$ constante.

\section{Aplicação da integral na determinação do sortimento de madeira}

A equação possibilitou classificar os sortimentos de madeira quanto ao volume, expresso em $\mathrm{m}^{3}$, e em número de peças de acordo com critérios estabelecidos para o planejamento da produção, permitindo ainda, quando desejado, a variação dos diâmetros e comprimentos de toras de acordo com a necessidade de planejamento ou da demanda de mercado.

Tomando por exemplo as dimensões de sortimentos: $\mathrm{S} 1=$ Madeira para celulose com diâmetro na ponta fina da tora entre 8 a $17,9 \mathrm{~cm}$ com casca e comprimento de 2,65 m; S2 = Madeira para celulose com diâmetro na ponta fina da tora entre 18 a 23,9 cm com casca e comprimento de 2,65 m; S3 = Madeira para serraria com diâmetro na ponta fina da tora entre 24 a $29,9 \mathrm{~cm}$ com casca e comprimento de 2,65 m; S4 = Madeira para serraria com diâmetro na ponta fina da tora entre 30 a $39,9 \mathrm{~cm}$ com casca e comprimento de $2,65 \mathrm{~m} \mathrm{e} \mathrm{S5}=$ Madeira para serraria/ laminação com diâmetro na ponta fina da tora maior que $40 \mathrm{~cm}$ com casca e comprimento de $2,65 \mathrm{~m}$ foi elaborada a Tabela de Sortimentos para Pinus taeda.

$\mathrm{Na}$ simulação do volume aproveitável em toras o menor diâmetro considerado foi de $8 \mathrm{~cm}$ com casca. Diâmetros iguais ou maiores que $24 \mathrm{~cm}$ com casca foram classificados como toras para serraria conforme esquema apresentado na Figura 1.

A definição da altura $\left(h_{\mathrm{i}}\right)$, altura do tronco onde se localizava um diâmetro de sortimento " $\mathrm{d}_{\mathrm{i}}$ ", foi estimada pela equação polinomial do quinto grau tendo como variável dependente $(\mathrm{h} / \mathrm{h})$ e como independente os diâmetros relativos $\left(\mathrm{d}_{\mathrm{d}} / \mathrm{d}\right)$.

Os resultados foram avaliados quanto à acurácia para a altura onde se localizavam os diâmetros de 24, 18 e $8 \mathrm{~cm}$ utilizando as estatísticas viés, média das diferenças absolutas $(m d)$ e desvio 


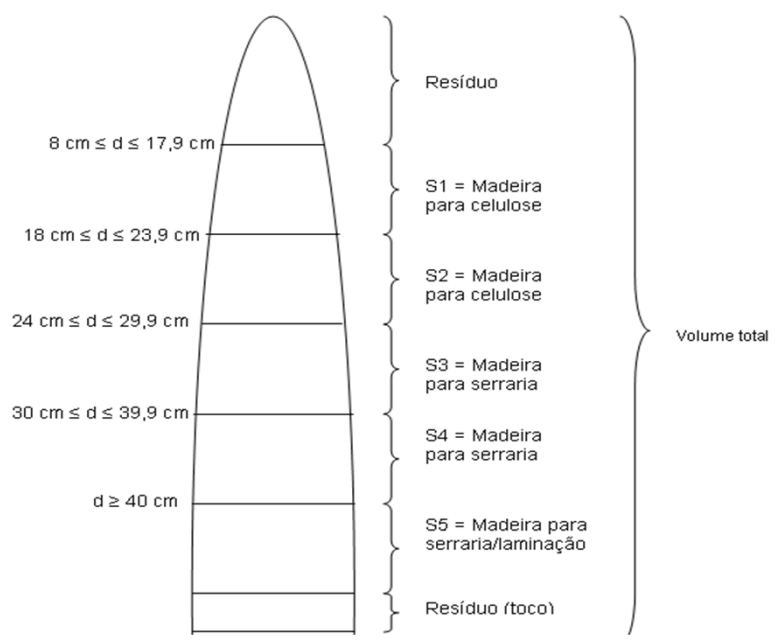

FIGURA 1: Sortimentos da árvore utilizados neste estudo.

FIGURE 1: Assortments found in the tree used in this study.

padrão das diferenças $(d p d)$. Essas medidas mostraram também a acurácia nas estimativas dos volumes dos sortimentos.

$\mathrm{Na}$ formação dos sortimentos foi dado preferência às toras de maior diâmetro, sendo o restante classificado nas classes imediatamente inferiores. A parte do fuste com diâmetro inferior a $8 \mathrm{~cm}$ e a parte que não possibilitou a formação de tora com comprimento de $2,65 \mathrm{~m}$ foi incluída como volume de resíduo.

$\mathrm{Na}$ construção da tabela de sortimento foi considerada a amplitude de diâmetro entre 12,0 e 44,0 cm., podendo-se alterar a apresentação de acordo com o interesse desde que, seja observada a amplitude dos dados observados.

Para a estimativa da altura total (h) correspondente ao diâmetro de entrada na tabela foi empregada uma equação de relação hipsométrica e definidas duas alturas localizadas em $\mathrm{h}-23 \% \mathrm{e} \mathrm{h}+23$ $\%$. Esses percentuais foram determinados após a análise das amplitudes das alturas amostradas em cada classe de dap e descreveram a variação natural observada entre as alturas em uma mesma classe de diâmetro.

Após a estimativa da altura total da árvore foi determinado o valor do quociente de forma e selecionados os coeficientes do polinômio do quinto grau para as classes de quocientes de forma $\left(\mathrm{K}_{0,7 \mathrm{~h}}\right)$ igual a 0,$4 ; 0,5$ e 0,6 .

A estimativa da altura por relação hipsométrica só ocorre na construção de uma tabela de sortimentos em decorrência da variação natural da altura de árvores com mesmo dap. Para fins de formação dos sortimentos em um sistema automatizado de inventário florestal ou de planejamento da produção a informação da altura e do diâmetro será levantada a campo.

Para a obtenção dos diâmetros com casca a partir dos diâmetros sem casca obtidos por análise de tronco, foi utilizada a expressão do fator de casca (K) calculada por Schneider et al., (2005), com $\mathrm{R}^{2}$ $\begin{array}{lllll}\text { de } & 0,96 \quad \text { e } & \text { CV\% } & \text { de } & 5,08 . \\ K=\sqrt{1-\frac{d^{2}}{\left(e x p^{0,27585+016105 d-0,00118 d^{2}}\right)}} & \text { e, a }\end{array}$ seguir o diâmetro com casca foi calculado por $\mathrm{dcc}=$ $\mathrm{dsc} / \mathrm{k}$.

\section{Validação das estimativas}

A validação dos modelos que estimam as variáveis: altura total (h), diâmetro a $70 \%$ da altura total $\left(\mathrm{d}_{0,7 \mathrm{~h}}\right)$ e volume da árvore foi efetuada com base no teste de Qui-quadrado $\left(\chi^{2}\right)$, com um nível de significância $(\alpha)$ de 0,01 , utilizando a equação:

$$
\chi^{2}=\sum_{i=1}^{n} \frac{(Y-\hat{Y})^{2}}{Y}
$$

Para essa validação foram usados dados de 18 árvores distribuídas na amplitude de diâmetros das árvores amostradas para o estudo e não utilizadas no desenvolvimento das equações.

\section{RESULTADOS E DISCUSSÕES}

\section{Equações para estimativa dos volumes dos fustes}

O ajuste do polinômio do quinto grau com dados não estratificados e estratificados em classes de quociente de forma, (Tabela 2), mostrou para qualquer dos casos coeficiente de determinação e de variação com valores acima de $98 \%$ e abaixo de $6 \%$, respectivamente. Tomando por base essas estatísticas pode-se afirmar que, independentemente do modo como os dados foram dispostos para o cálculo, houve bom ajuste e precisão em todas as situações.

A comparação estatística entre dados estratificados ou não estratificados (Tabela 3) mostrou resultado semelhante. De modo geral não ocorreram tendências e os valores estimados foram próximos dos observados. Entretanto, a estratificação em classe de quociente de forma 
TABELA 2: Estatísticas das equações ajustadas para estimativa dos volumes dos fustes.

TABLE 2: Statistics of adjusted equations to estimate the volumes of the bole.

Parâmetros estimados

\begin{tabular}{ccccccccc}
\hline $\begin{array}{c}\text { Critérios de } \\
\text { estratificação }\end{array}$ & $\hat{\beta}_{0}$ & $\hat{\beta}_{1}$ & $\hat{\beta}_{2}$ & $\hat{\beta}_{3}$ & $\hat{\beta}_{4}$ & $\hat{\beta}_{5}$ & $R^{2}(\%)$ & CV(\%) \\
\hline s/estratificação & 1,1554 & $-2,7308$ & 11,8784 & $-26,2512$ & 24,0259 & $-8,0693$ & 98,5 & 5,7 \\
$\mathrm{~K}_{0,7 \mathrm{~h}} 0,4$ & 1,1729 & $-2,9716$ & 12,8409 & $-29,0849$ & 27,3410 & $-9,2678$ & 99,0 & 4,9 \\
$\mathrm{~K}_{0,7 \mathrm{~h}} 0,5$ & 1,1585 & $-2,7318$ & 12,0225 & $-26,4724$ & 24,0633 & $-8,0297$ & 99,0 & 4,7 \\
$\mathrm{~K}_{0,7 \mathrm{~h}} 0,6$ & 1,1116 & $-2,2815$ & 10,0144 & $-21,2078$ & 18,5924 & $-6,2600$ & 98,5 & 5,2 \\
\hline
\end{tabular}

Em que: Todos os coeficientes são significativos com $(\mathrm{p} \leq 0,05)$.

TABELA 3: Estatísticas viés (v), média das diferenças absolutas (md) e desvio padrão das diferenças (dpd) e o ranking para a estimativa dos volumes.

TABLE 3: Statistical bias (v), mean absolute differences (md) and standard deviation of differences (dpd) and ranking for the estimation of volumes.

\begin{tabular}{lllll}
\hline $\begin{array}{c}\text { Critérios de } \\
\text { estratificação }\end{array}$ & $v$ & md & dpd total \\
\hline
\end{tabular}

s/estratificação -0,0157 (2) 0,0348 (2) 0,0614 (2) 06

Classes $\mathrm{K}_{0,7 \mathrm{~h}} \quad-0,0117$ (1) 0,0333 (1) 0,0578 (1) 03

trouxe ganho ao modelo, resultando em melhores estimativas em relação às obtidas com dados não estratificados.

A Figura 2 confirma esse resultado com o modelo calculado com os dados não estratificados, de forma geral com bom desempenho, apresentou leve superestimava do volume para as árvores com dap entre 20 e $35 \mathrm{~cm}$, enquanto o modelo ajustado com os dados estratificados não mostrou a mesma tendência e apresentando ainda menor dispersão dos valores residuais.

O mesmo resultado também foi observado por Assis et al. (2001) que comparando o desempenho dos modelos de afilamento de Clark et al. (1991), Max e Burkhart (1976), Hradetzky (1976) e Goulding e Murray (1976), com relação à estimativa de volumes totais e parciais para a espécie Pinus taeda L., também encontraram evidencias da melhoria nas estimativas quando da classificação dos dados amostrais buscando a redução da variabilidade total. Os autores afirmaram que a classificação dos dados em classes de dap permitiu o uso dos quatro modelos com segurança.
A partir deste resultado que indicou a estratificação dos dados com base na classe de quociente de forma $\mathrm{K}_{0,7 \mathrm{~h}}$ melhorou o ajuste do modelo, este procedimento foi adotado para a construção da tabela de sortimentos (Tabela 4).

$\mathrm{Na}$ tabela os sortimentos foram apresentados para diâmetros entre 12 e $44 \mathrm{~cm}$ e três alturas para cada diâmetro, que representam a variação na relação hipsométrica, o valor do quociente de forma $\left(\mathrm{K}_{0,7 \mathrm{~h}}\right)$, os sortimentos $\mathrm{S} 1$, $\mathrm{S} 2, \mathrm{~S} 3, \mathrm{~S} 4$ e S5 com número de toras (n) e em percentagem do volume total encontrado na classe, o volume do resíduo expresso $\mathrm{em}^{3} \mathrm{e}$ em percentagem do volume total, bem como o volume total $\mathrm{em}^{3}$, para cada árvore, obtido com a integração do polinômio segundo os parâmetros calculados com dados estratificados em classes de quocientes de forma.

Os dados da tabela mostraram a redução da percentagem de volume de madeira classificada como resíduos, assim como o deslocamento dos sortimentos para as maiores classes quando do aumento das dimensões de dap e $\mathrm{h}$ da árvore.

As equações calculadas permitem, caso necessário, variar as dimensões de diâmetro e comprimento das toras segundo os critérios de planejamento da empresa, alterando os valores de número de toras e de volume nas classes de sortimento, porém mantendo a acurácia das estimativas.

$\mathrm{Na}$ determinação da altura do tronco onde se localizava o diâmetro mínimo desejado para um sortimento, no presente caso 24,18 e $8 \mathrm{~cm}$, a equação do polinômio do quinto grau re-estimada, contendo a variável dependente (hi/h) e independentes (di/d), forneceu estimativas acuradas conforme mostrado nas estatísticas da Tabela 5 . 
Se m e stratificação dos dados

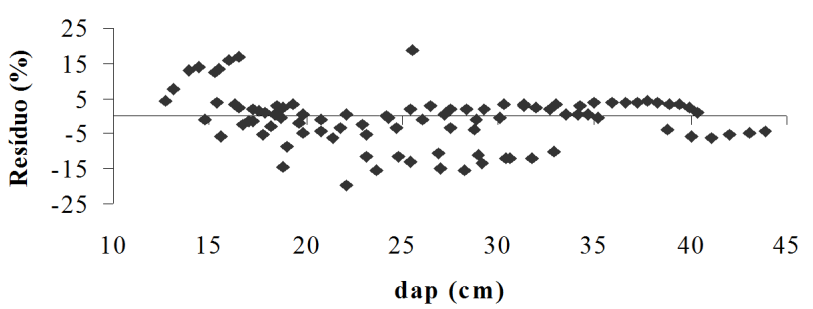

Com es tratifição dos dados

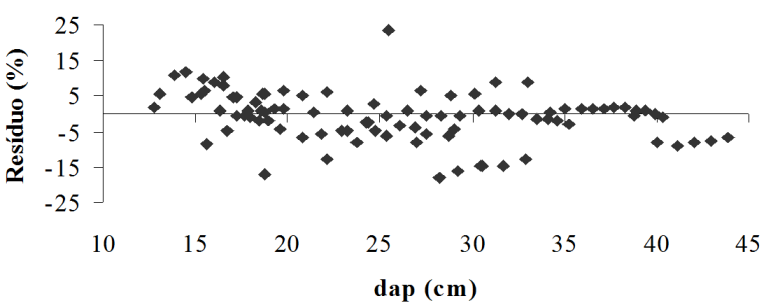

FIGURA 2: Distribuição dos resíduos da estimativa do volume total a partir dos dados sem e com estratificação.

FIGURE 2: Distribution of residues of the estimated total volume from the data with and without stratification.

TABELA 4: Sortimentos para serraria e celulose de Pinus taeda L.

TABLE 4: Assortment for sawmill and pulp of Pinus taeda L.

\begin{tabular}{|c|c|c|c|c|c|c|c|c|c|c|c|c|c|c|c|}
\hline \multirow{2}{*}{$\begin{array}{l}\text { dap } \\
(\mathrm{cm})\end{array}$} & \multirow{2}{*}{$\begin{array}{c}\mathrm{h} \\
(\mathrm{m})\end{array}$} & \multirow{2}{*}{$\frac{d_{0,7 h}}{d a p}$} & \multicolumn{2}{|c|}{$\mathrm{S} 1$} & \multicolumn{2}{|c|}{$\mathrm{S} 2$} & \multicolumn{2}{|c|}{ S3 } & \multicolumn{2}{|c|}{$\mathrm{S} 4$} & \multicolumn{2}{|c|}{ S5 } & \multirow{2}{*}{$\begin{array}{l}\text { V Res } \\
\left(\mathrm{m}^{3} \mathrm{~s} / \mathrm{c}\right)\end{array}$} & \multirow{2}{*}{$\%$} & \multirow{2}{*}{$\begin{array}{l}V \text { total } \\
\left(\mathrm{m}^{3} \mathrm{~s} / \mathrm{c}\right)\end{array}$} \\
\hline & & & $\mathrm{n}$ & $\%$ & $\mathrm{n}$ & $\%$ & $\mathrm{n}$ & $\%$ & $\mathrm{n}$ & $\%$ & $\mathrm{n}$ & $\%$ & & & \\
\hline 12 & 5,9 & 0,4 & 1 & 83,5 & 0 & 0 & 0 & 0 & 0 & 0 & 0 & 0 & 0,0050 & 16,5 & 0,0305 \\
\hline 12 & 7,0 & 0,4 & 1 & 70,1 & 0 & 0 & 0 & 0 & 0 & 0 & 0 & 0 & 0,0108 & 29,9 & 0,0361 \\
\hline 12 & 8,0 & 0,4 & 1 & 63,0 & 0 & 0 & 0 & 0 & 0 & 0 & 0 & 0 & 0,0155 & 37,0 & 0,0418 \\
\hline 13 & 6,4 & 0,4 & 1 & 74,2 & 0 & 0 & 0 & 0 & 0 & 0 & 0 & 0 & 0,0101 & 25,8 & 0,0391 \\
\hline 13 & 7,8 & 0,4 & 1 & 64,0 & 0 & 0 & 0 & 0 & 0 & 0 & 0 & 0 & 0,0173 & 36,0 & 0,0480 \\
\hline 13 & 9,3 & 0,4 & 1 & 56,1 & 0 & 0 & 0 & 0 & 0 & 0 & 0 & 0 & 0,0250 & 43,9 & 0,0570 \\
\hline 14 & 7,0 & 0,4 & 1 & 69,5 & 0 & 0 & 0 & 0 & 0 & 0 & 0 & 0 & 0,0151 & 30,5 & 0,0497 \\
\hline 14 & 8,8 & 0,4 & 1 & 58,5 & 0 & 0 & 0 & 0 & 0 & 0 & 0 & 0 & 0,0260 & 41,5 & 0,0626 \\
\hline 14 & 10,5 & 0,4 & 2 & 83,9 & 0 & 0 & 0 & 0 & 0 & 0 & 0 & 0 & 0,0122 & 16,1 & 0,0756 \\
\hline 15 & 7,8 & 0,4 & 1 & 64,1 & 0 & 0 & 0 & 0 & 0 & 0 & 0 & 0 & 0,0229 & 35,9 & 0,0638 \\
\hline 15 & 9,8 & 0,4 & 2 & 87,4 & 0 & 0 & 0 & 0 & 0 & 0 & 0 & 0 & 0,0101 & 12,6 & 0,0803 \\
\hline 15 & 11,7 & 0,4 & 2 & 78,5 & 0 & 0 & 0 & 0 & 0 & 0 & 0 & 0 & 0,0208 & 21,5 & 0,0968 \\
\hline 16 & 8,3 & 0,4 & 2 & 93,8 & 0 & 0 & 0 & 0 & 0 & 0 & 0 & 0 & 0,0048 & 6,2 & 0,0773 \\
\hline 16 & 10,8 & 0,4 & 2 & 82,7 & 0 & 0 & 0 & 0 & 0 & 0 & 0 & 0 & 0,0175 & 17,3 & 0,1012 \\
\hline 16 & 13,3 & 0,4 & 3 & 91,4 & 0 & 0 & 0 & 0 & 0 & 0 & 0 & 0 & 0,0108 & 8,6 & 0,1251 \\
\hline 17 & 9,1 & 0,4 & 2 & 90,4 & 0 & 0 & 0 & 0 & 0 & 0 & 0 & 0 & 0,0093 & 9,6 & 0,0960 \\
\hline 17 & 11,8 & 0,4 & 2 & 78,0 & 0 & 0 & 0 & 0 & 0 & 0 & 0 & 0 & 0,0276 & 22,0 & 0,1256 \\
\hline 17 & 14,6 & 0,4 & 3 & 87,5 & 0 & 0 & 0 & 0 & 0 & 0 & 0 & 0 & 0,0193 & 12,5 & 0,1552 \\
\hline 18 & 9,9 & 0,4 & 2 & 86,7 & 0 & 0 & 0 & 0 & 0 & 0 & 0 & 0 & 0,0156 & 13,3 & 0,1175 \\
\hline 18 & 12,9 & 0,4 & 3 & 92,5 & 0 & 0 & 0 & 0 & 0 & 0 & 0 & 0 & 0,0116 & 7,5 & 0,1536 \\
\hline 18 & 15,9 & 0,5 & 4 & 93,8 & 0 & 0 & 0 & 0 & 0 & 0 & 0 & 0 & 0,0128 & 6,2 & 0,2068 \\
\hline 19 & 10,7 & 0,4 & 2 & 83,0 & 0 & 0 & 0 & 0 & 0 & 0 & 0 & 0 & 0,0242 & 17,0 & 0,1419 \\
\hline 19 & 14,0 & 0,4 & 3 & 89,4 & 0 & 0 & 0 & 0 & 0 & 0 & 0 & 0 & 0,0196 & 10,6 & 0,1854 \\
\hline 19 & 17,2 & 0,5 & 3 & 59,7 & 1 & 31,0 & 0 & 0 & 0 & 0 & 0 & 0 & 0,0231 & 9,3 & 0,2495 \\
\hline 20 & 11,5 & 0,4 & 3 & 95,9 & 0 & 0 & 0 & 0 & 0 & 0 & 0 & 0 & 0,0069 & 4,1 & 0,1693 \\
\hline 20 & 15,0 & 0,4 & 3 & 58,5 & 1 & 37,8 & 0 & 0 & 0 & 0 & 0 & 0 & 0,0082 & 3,7 & 0,2211 \\
\hline 20 & 18,4 & 0,5 & 4 & 66,9 & 1 & 29,2 & 0 & 0 & 0 & 0 & 0 & 0 & 0,0116 & 3,9 & 0,2974 \\
\hline 21 & 12,3 & 0,4 & 2 & 49,6 & 1 & 44,4 & 0 & 0 & 0 & 0 & 0 & 0 & 0,0120 & 6,0 & 0,1998 \\
\hline 21 & 16,0 & 0,4 & 3 & 58,7 & 1 & 35,8 & 0 & 0 & 0 & 0 & 0 & 0 & 0,0145 & 5,5 & 0,2608 \\
\hline 21 & 19,7 & 0,5 & 3 & 34,2 & 2 & 49,5 & 0 & 0 & 0 & 0 & 0 & 0 & 0,0569 & 16,2 & 0,3507 \\
\hline 22 & 13,1 & 0,4 & 2 & 49,7 & 1 & 42,2 & 0 & 0 & 0 & 0 & 0 & 0 & 0,0190 & 8,1 & 0,2333 \\
\hline 22 & 17,0 & 0,4 & 2 & 33,0 & 2 & 59,7 & 0 & 0 & 0 & 0 & 0 & 0 & 0,0221 & 7,2 & 0,3045 \\
\hline 22 & 20,9 & 0,5 & 3 & 31,7 & 3 & 65,7 & 0 & 0 & 0 & 0 & 0 & 0 & 0,0103 & 2,5 & 0,4093 \\
\hline
\end{tabular}

Continua ... 
TABELA 4: Continuação ...

TABLE 4: Continued ...

\begin{tabular}{|c|c|c|c|c|c|c|c|c|c|c|c|c|c|c|c|}
\hline \multirow{2}{*}{$\begin{array}{l}\text { dap } \\
(\mathrm{cm})\end{array}$} & \multirow{2}{*}{$\begin{array}{c}\mathrm{h} \\
(\mathrm{m})\end{array}$} & \multirow{2}{*}{$\frac{d_{0,7 h}}{d a p}$} & \multicolumn{2}{|c|}{$\mathrm{S} 1$} & \multicolumn{2}{|c|}{$\mathrm{S} 2$} & \multicolumn{2}{|c|}{ S3 } & \multicolumn{2}{|c|}{ S4 } & \multicolumn{2}{|c|}{ S5 } & \multirow{2}{*}{$\begin{array}{l}\text { V Res } \\
\left(\mathrm{m}^{3} \mathrm{~s} / \mathrm{c}\right)\end{array}$} & \multirow{2}{*}{$\%$} & \multirow{2}{*}{$\begin{array}{l}\mathrm{V} \text { total } \\
\left(\mathrm{m}^{3} \mathrm{~s} / \mathrm{c}\right)\end{array}$} \\
\hline & & & $\mathrm{n}$ & $\%$ & $\mathrm{n}$ & $\%$ & $\mathrm{n}$ & $\%$ & $\mathrm{n}$ & $\%$ & $\mathrm{n}$ & $\%$ & & & \\
\hline 23 & 13,9 & 0,4 & 2 & 49,4 & 1 & 40,3 & 0 & 0 & 0 & 0 & 0 & 0 & 0,0279 & 10,3 & 0,2700 \\
\hline 23 & 18,0 & 0,4 & 3 & 40,2 & 2 & 57,1 & 0 & 0 & 0 & 0 & 0 & 0 & 0,0092 & 2,6 & 0,3523 \\
\hline 23 & 22,1 & 0,5 & 3 & 33,2 & 3 & 62,9 & 0 & 0 & 0 & 0 & 0 & 0 & 0,0187 & 4,0 & 0,4734 \\
\hline 24 & 14,6 & 0,4 & 2 & 29,9 & 2 & 67,3 & 0 & 0 & 0 & 0 & 0 & 0 & 0,0089 & 2,9 & 0,3098 \\
\hline 24 & 19,0 & 0,4 & 3 & 41,4 & 2 & 54,8 & 0 & 0 & 0 & 0 & 0 & 0 & 0,0153 & 3,8 & 0,4041 \\
\hline 24 & 23,3 & 0,5 & 2 & 19,1 & 4 & 75,3 & 0 & 0 & 0 & 0 & 0 & 0 & 0,0302 & 5,6 & 0,5430 \\
\hline 25 & 15,3 & 0,4 & 2 & 31,1 & 2 & 64,9 & 0 & 0 & 0 & 0 & 0 & 0 & 0,0140 & 4,0 & 0,3527 \\
\hline 25 & 19,9 & 0,4 & 2 & 22,8 & 2 & 42,2 & 1 & 29,9 & 0 & 0 & 0 & 0 & 0,0233 & 5,1 & 0,4600 \\
\hline 25 & 24,4 & 0,5 & 3 & 24,7 & 3 & 49,9 & 1 & 22,9 & 0 & 0 & 0 & 0 & 0,0157 & 2,5 & 0,6180 \\
\hline 26 & 16,0 & 0,4 & 1 & 20,5 & 1 & 26,9 & 1 & 35,9 & 0 & 0 & 0 & 0 & 0,0669 & 16,8 & 0,3987 \\
\hline 26 & 20,8 & 0,5 & 2 & 16,1 & 3 & 55,2 & 1 & 26,4 & 0 & 0 & 0 & 0 & 0,0132 & 2,3 & 0,5664 \\
\hline 26 & 25,5 & 0,5 & 2 & 14,0 & 3 & 42,5 & 2 & 39,8 & 0 & 0 & 0 & 0 & 0,0254 & 3,6 & 0,6984 \\
\hline 27 & 16,6 & 0,4 & 1 & 12,3 & 2 & 46,6 & 1 & 34,7 & 0 & 0 & 0 & 0 & 0,0289 & 6,4 & 0,4478 \\
\hline 27 & 21,6 & 0,5 & 2 & 17,3 & 2 & 33,6 & 2 & 45,9 & 0 & 0 & 0 & 0 & 0,0208 & 3,3 & 0,6361 \\
\hline 27 & 26,6 & 0,5 & 2 & 18,2 & 3 & 41,8 & 2 & 38,5 & 0 & 0 & 0 & 0 & 0,0122 & 1,6 & 0,7842 \\
\hline 28 & 17,3 & 0,4 & 2 & 18,8 & 2 & 45,7 & 1 & 33,6 & 0 & 0 & 0 & 0 & 0,0092 & 1,8 & 0,5000 \\
\hline 28 & 22,4 & 0,5 & 1 & 6,9 & 3 & 44,3 & 2 & 44,4 & 0 & 0 & 0 & 0 & 0,0305 & 4,3 & 0,7101 \\
\hline 28 & 27,6 & 0,5 & 2 & 10,2 & 3 & 35,1 & 3 & 52,5 & 0 & 0 & 0 & 0 & 0,0199 & 2,3 & 0,8754 \\
\hline 29 & 17,9 & 0,4 & 2 & 19,9 & 1 & 20,1 & 2 & 57,5 & 0 & 0 & 0 & 0 & 0,0136 & 2,4 & 0,5553 \\
\hline 29 & 23,2 & 0,5 & 2 & 11,3 & 2 & 26,6 & 3 & 60,6 & 0 & 0 & 0 & 0 & 0,0119 & 1,5 & 0,7885 \\
\hline 29 & 28,5 & 0,5 & 2 & 11,0 & 2 & 21,4 & 4 & 64,6 & 0 & 0 & 0 & 0 & 0,0297 & 3,1 & 0,9719 \\
\hline 30 & 18,4 & 0,4 & 2 & 20,9 & 1 & 19,9 & 2 & 56,1 & 0 & 0 & 0 & 0 & 0,0191 & 3,1 & 0,6136 \\
\hline 30 & 23,9 & 0,5 & 2 & 12,3 & 2 & 26,6 & 3 & 59,0 & 0 & 0 & 0 & 0 & 0,0182 & 2,1 & 0,8712 \\
\hline 30 & 29,4 & 0,5 & 3 & 14,3 & 2 & 21,4 & 3 & 43,5 & 1 & 19,5 & 0 & 0 & 0,0141 & 1,3 & 1,0737 \\
\hline 31 & 19,0 & 0,4 & 1 & 7,7 & 2 & 33,7 & 2 & 54,8 & 0 & 0 & 0 & 0 & 0,0257 & 3,8 & 0,6750 \\
\hline 31 & 24,6 & 0,5 & 2 & 13,1 & 1 & 11,8 & 3 & 49,6 & 1 & 22,8 & 0 & 0 & 0,0262 & 2,7 & 0,9582 \\
\hline 31 & 30,3 & 0,5 & 2 & 7,9 & 2 & 17,1 & 4 & 54,1 & 1 & 19,0 & 0 & 0 & 0,0215 & 1,8 & 1,1809 \\
\hline 32 & 19,5 & 0,4 & 2 & 11,7 & 1 & 14,2 & 3 & 42,7 & 1 & 30,4 & 0 & 0 & 0,0074 & 1,0 & 0,7394 \\
\hline 32 & 25,3 & 0,5 & 2 & 7,8 & 2 & 20,5 & 3 & 48,6 & 1 & 22,2 & 0 & 0 & 0,0089 & 0,8 & 1,0495 \\
\hline 32 & 31,2 & 0,5 & 2 & 8,6 & 2 & 17,3 & 3 & 38,0 & 2 & 33,7 & 0 & 0 & 0,0307 & 2,4 & 1,2934 \\
\hline 33 & 20,0 & 0,4 & 2 & 12,5 & 1 & 14,4 & 2 & 42,0 & 1 & 29,8 & 0 & 0 & 0,0107 & 1,3 & 0,8069 \\
\hline 33 & 26,0 & 0,5 & 2 & 8,5 & 2 & 20,8 & 2 & 30,3 & 2 & 39,2 & 0 & 0 & 0,0137 & 1,2 & 1,1451 \\
\hline 33 & 32,0 & 0,5 & 2 & 5,6 & 2 & 13,3 & 4 & 47,2 & 2 & 33,0 & 0 & 0 & 0,0138 & 1,0 & 1,4112 \\
\hline 34 & 20,5 & 0,4 & 2 & 13,3 & 1 & 14,5 & 2 & 41,4 & 1 & 29,2 & 0 & 0 & 0,0147 & 1,7 & 0,8775 \\
\hline 34 & 26,6 & 0,5 & 2 & 9,2 & 1 & 9,0 & 3 & 41,7 & 2 & 38,5 & 0 & 0 & 0,0197 & 1,6 & 1,2452 \\
\hline 34 & 32,7 & 0,5 & 2 & 6,1 & 2 & 13,7 & 3 & 33,4 & 3 & 45,5 & 0 & 0 & 0,0203 & 1,3 & 1,5344 \\
\hline 35 & 20,9 & 0,4 & 1 & 4,7 & 2 & 23,9 & 1 & 18,8 & 2 & 50,6 & 0 & 0 & 0,0195 & 2,0 & 0,9511 \\
\hline 35 & 27,2 & 0,5 & 1 & 3,6 & 2 & 15,4 & 2 & 25,9 & 3 & 53,1 & 0 & 0 & 0,0269 & 2,0 & 1,3496 \\
\hline 35 & 33,5 & 0,5 & 2 & 6,6 & 2 & 14,0 & 2 & 20,9 & 4 & 56,7 & 0 & 0 & 0,0283 & 1,7 & 1,6630 \\
\hline 36 & 21,4 & 0,4 & 1 & 5,0 & 1 & 9,6 & 2 & 33,2 & 2 & 49,8 & 0 & 0 & 0,0251 & 2,4 & 1,0280 \\
\hline 36 & 27,8 & 0,5 & 2 & 5,7 & 2 & 15,8 & 2 & 25,7 & 3 & 52,1 & 0 & 0 & 0,0084 & 0,6 & 1,4585 \\
\hline 36 & 34,2 & 0,5 & 3 & 8,6 & 1 & 6,2 & 3 & 28,9 & 4 & 55,7 & 0 & 0 & 0,0116 & 0,6 & 1,7971 \\
\hline 37 & 21,8 & 0,4 & 1 & 5,4 & 1 & 9,8 & 2 & 33,0 & 2 & 49,0 & 0 & 0 & 0,0315 & 2,8 & 1,1081 \\
\hline 37 & 28,3 & 0,5 & 2 & 6,3 & 1 & 6,7 & 2 & 21,3 & 4 & 64,9 & 0 & 0 & 0,0124 & 0,8 & 1,5720 \\
\hline 37 & 34,9 & 0,5 & 2 & 4,6 & 2 & 10,9 & 2 & 17,9 & 5 & 65,7 & 0 & 0 & 0,0169 & 0,9 & 1,9369 \\
\hline 38 & 22,2 & 0,4 & 2 & 8,2 & 1 & 10,1 & 1 & 14,6 & 3 & 66,4 & 0 & 0 & 0,0091 & 0,8 & 1,1915 \\
\hline 38 & 28,9 & 0,5 & 2 & 6,7 & 1 & 6,9 & 2 & 21,4 & 4 & 63,9 & 0 & 0 & 0,0175 & 1,0 & 1,6902 \\
\hline 38 & 35,5 & 0,5 & 2 & 9,8 & 0 & 0,0 & 3 & 24,4 & 5 & 64,7 & 0 & 0 & 0,0236 & 1,1 & 2,0825 \\
\hline 39 & 22,7 & 0,4 & 2 & 8,2 & 1 & 10,3 & 1 & 14,6 & 3 & 65,5 & 0 & 0 & 0,0185 & 1,5 & 1,2783 \\
\hline 39 & 29,4 & 0,5 & 2 & 7,2 & 1 & 7,1 & 1 & 9,6 & 5 & 74,8 & 0 & 0 & 0,0236 & 1,3 & 1,8132 \\
\hline 39 & 36,2 & 0,5 & 2 & 5,4 & 1 & 4,9 & 2 & 14,8 & 6 & 73,4 & 0 & 0 & 0,0315 & 1,4 & 2,2340 \\
\hline
\end{tabular}

Continua ... 
TABELA 4: Continuação ...

TABLE 4: Continued ...

\begin{tabular}{|c|c|c|c|c|c|c|c|c|c|c|c|c|c|c|c|}
\hline \multirow{2}{*}{$\begin{array}{l}\text { dap } \\
(\mathrm{cm})\end{array}$} & \multirow{2}{*}{$\begin{array}{c}\mathrm{h} \\
(\mathrm{m})\end{array}$} & \multirow{2}{*}{$\frac{d_{0,7 h}}{d a p}$} & \multicolumn{2}{|c|}{$\mathrm{S} 1$} & \multicolumn{2}{|c|}{$\mathrm{S} 2$} & \multicolumn{2}{|c|}{ S3 } & \multicolumn{2}{|c|}{$\mathrm{S} 4$} & \multicolumn{2}{|c|}{ S5 } & \multirow{2}{*}{$\begin{array}{l}\text { V Res } \\
\left(\mathrm{m}^{3} \mathrm{~s} / \mathrm{c}\right)\end{array}$} & \multirow{2}{*}{$\%$} & \multirow{2}{*}{$\begin{array}{l}\mathrm{V} \text { total } \\
\left(\mathrm{m}^{3} \mathrm{~s} / \mathrm{c}\right)\end{array}$} \\
\hline & & & $\mathrm{n}$ & $\%$ & $\mathrm{n}$ & $\%$ & $\mathrm{n}$ & $\%$ & $\mathrm{n}$ & $\%$ & $\mathrm{n}$ & $\%$ & & & \\
\hline 40 & 23,0 & 0,5 & 1 & 3,8 & 1 & 7,4 & 1 & 11,5 & 4 & 76,0 & 0 & 0 & 0,0209 & 1,4 & 1,4908 \\
\hline 40 & 29,9 & 0,5 & 1 & 2,8 & 1 & 4,8 & 2 & 16,9 & 4 & 54,6 & 1 & 19,2 & 0,0308 & 1,6 & 1,9412 \\
\hline 40 & 36,8 & 0,5 & 2 & 3,4 & 2 & 8,6 & 2 & 15,0 & 5 & 56,4 & 1 & 16,0 & 0,0125 & 0,5 & 2,3916 \\
\hline 41 & 23,4 & 0,5 & 1 & 4,0 & 1 & 7,6 & 1 & 11,6 & 3 & 51,3 & 1 & 23,8 & 0,0270 & 1,7 & 1,5932 \\
\hline 41 & 30,4 & 0,5 & 2 & 4,5 & 1 & 5,0 & 2 & 17,1 & 4 & 54,0 & 1 & 19,0 & 0,0093 & 0,4 & 2,0744 \\
\hline 41 & 37,4 & 0,6 & 1 & 1,9 & 2 & 7,9 & 2 & 13,7 & 6 & 61,7 & 1 & 13,9 & 0,0241 & 0,9 & 2,6601 \\
\hline 42 & 23,8 & 0,5 & 1 & 4,3 & 1 & 7,8 & 1 & 11,7 & 3 & 50,7 & 1 & 23,4 & 0,0340 & 2,0 & 1,6996 \\
\hline 42 & 30,9 & 0,5 & 1 & 4,8 & 1 & 5,2 & 1 & 7,5 & 4 & 47,9 & 2 & 33,9 & 0,0133 & 0,6 & 2,2129 \\
\hline 42 & 38,1 & 0,6 & 1 & 2,1 & 1 & 3,4 & 2 & 11,1 & 6 & 56,8 & 2 & 25,4 & 0,0334 & 1,2 & 2,8377 \\
\hline 43 & 24,2 & 0,5 & 2 & 6,5 & 0 & 0 & 2 & 19,8 & 3 & 50,2 & 1 & 23,1 & 0,0071 & 0,4 & 1,8105 \\
\hline 43 & 31,4 & 0,5 & 1 & 5,2 & 1 & 5,4 & 1 & 7,6 & 4 & 47,6 & 2 & 33,4 & 0,0182 & 0,8 & 2,3571 \\
\hline 43 & 38,7 & 0,6 & 2 & 3,0 & 1 & 3,6 & 2 & 11,3 & 5 & 46,0 & 3 & 35,4 & 0,0217 & 0,7 & 3,0225 \\
\hline 44 & 24,6 & 0,5 & 2 & 7,0 & 0 & 0 & 1 & 8,2 & 3 & 43,2 & 2 & 41,1 & 0,0102 & 0,5 & 1,9258 \\
\hline 44 & 31,9 & 0,5 & 1 & 2,0 & 1 & 3,6 & 2 & 13,3 & 4 & 47,2 & 2 & 33,0 & 0,0243 & 1,0 & 2,5072 \\
\hline 44 & 39,3 & 0,6 & 2 & 3,8 & 1 & 3,8 & 2 & 11,5 & 5 & 45,6 & 3 & 34,9 & 0,0157 & 0,5 & 3,2148 \\
\hline
\end{tabular}

Em que: dap = Diâmetro à altura do peito $(1,30 \mathrm{~m})$, em centímetros; $\mathrm{h}=$ Altura total da árvore, com três valores para cada diâmetro, em metros; d0,7h/dap = Quociente de forma; S1; S2; S3; S4 e S5 = Classes de sortimentos; $\mathrm{n}=$ Numero de toras; $\%=$ Percentagem do volume de cada sortimento e do resíduo; $\mathrm{V}$ res $=$ Volume do resíduo $\left(\mathrm{m}^{3}\right)$ e $\mathrm{V}$ total $=$ Volume total $\left(\mathrm{m}^{3}\right)$.

TABELA 5: Viés (v), média das diferenças absolutas (md) e desvio padrão das diferenças (dpd) para altura onde o diâmetro alcança 24,18 e $8 \mathrm{~cm}$ calculado com dados amostrais distribuídos em classes de quociente de forma $\mathrm{K}_{0,7 \mathrm{~h}^{\circ}}$.

TABLE 5: Bias (v), mean absolute of differences (md) and standard deviations of differences (dpd) of the height where the diameter reach 24,18 and 8 $\mathrm{cm}$ calculated with sampling data distributed in form quotient classes.

\begin{tabular}{cccc}
\hline $\mathrm{d}(\mathrm{cm})$ & viés & $\mathrm{md}$ & $\mathrm{dpd}$ \\
8 & 0,0018 & 0,2946 & 0,3743 \\
18 & $-0,0114$ & 0,4247 & 0,5316 \\
24 & 0,0012 & 0,4899 & 0,6696 \\
\hline
\end{tabular}

Os resultados mostraram que as estimativas das alturas foram muito próximas das observadas permitindo inferir sobre a qualidade dos volumes parciais do tronco com igual acurácia, visto que o volume das secções foi obtido pela integração da função de regressão multiplicada pela constante $\Pi / 4$.
Validações das equações para estimativas de $h$, $\mathrm{d}_{\mathbf{0}, \mathrm{h}}$ e volume

Os valores dos resíduos mostraram o bom ajuste obtido com as equações, dentro do limite das variáveis observadas. Apesar da eficiência das equações já ter sido comprovada, os resultados da Tabela 6 confirmaram, mais uma vez, a boa precisão alcançada com a estratificação dos dados amostrados em classes de quociente de forma.

$O$ teste de Qui-quadrado $\left(\chi^{2}\right)$, aplicado para comparar os valores observados e estimados nas diferentes dimensões das árvores (Tabela 7), confirmou não haver diferença significativa para a probabilidade de 0,01 para todas as variáveis testadas. $\mathrm{O}$ valor de $\chi^{2}$ calculado foi igual a 2,60 , 1,88 e 0,02 para as comparações entre valores observados e estimados da altura, diâmetro a $70 \%$ da altura $\left(\mathrm{d}_{0,7 \mathrm{~h}}\right)$ e volume, respectivamente; todos menores que o valor tabelado, com 17 graus de liberdade, 33,41.

Esse resultado também foi observado graficamente na Figura 3, que representa os valores observados e estimados de altura total, $\mathrm{d}_{0,7 \mathrm{~h}}$ e volume, onde foi possível verificar a quase total, ou total, sobreposição dos valores no caso dos volumes. 
TABELA 6: Validação das equações usadas para construção da tabela de sortimentos.

TABLE 6: Validation of the equations used to construct the assortment table.

\begin{tabular}{cccccccccc}
\hline $\begin{array}{c}\text { dap } \\
(\mathrm{cm})\end{array}$ & $\begin{array}{c}\mathrm{h} \\
(\mathrm{m})\end{array}$ & $\begin{array}{c}\mathrm{d}_{0,7 \mathrm{~h}} \\
(\mathrm{~cm})\end{array}$ & $\begin{array}{c}\text { Vol } \\
\left(\mathrm{m}^{3} \mathrm{~s} / \mathrm{c}\right)\end{array}$ & $\begin{array}{c}\mathrm{h}_{\text {est }} \\
(\mathrm{m})\end{array}$ & $\begin{array}{c}\text { resíduo } \\
(\%)\end{array}$ & $\begin{array}{c}\mathrm{d}_{0,7 \mathrm{~h} \text { est }} \\
(\mathrm{cm})\end{array}$ & $\begin{array}{c}\text { resíduo } \\
(\%)\end{array}$ & $\begin{array}{c}\text { vol est } \\
\left(\mathrm{m}^{3} \mathrm{~s} / \mathrm{c}\right)\end{array}$ & $\begin{array}{c}\text { resíduo } \\
(\%)\end{array}$ \\
\hline 14,8 & 12,1 & 6,6 & 0,1048 & 12,1 & $-0,1$ & 6,5 & 1,6 & 0,0996 & 4,9 \\
15,4 & 12,8 & 7,5 & 0,1264 & 12,6 & 1,9 & 6,8 & 9,1 & 0,1241 & 1,8 \\
16,3 & 14,4 & 7,9 & 0,1580 & 13,2 & 8,1 & 7,4 & 6,3 & 0,1564 & 1,0 \\
17,0 & 13,5 & 7,6 & 0,1539 & 13,8 & $-1,9$ & 7,3 & 4,1 & 0,1466 & 4,8 \\
18,2 & 15,1 & 8,0 & 0,1939 & 14,6 & 3,0 & 8,0 & 0,3 & 0,1879 & 3,1 \\
19,3 & 19,8 & 10,0 & 0,3054 & 15,5 & 21,9 & 9,6 & 3,5 & 0,3016 & 1,2 \\
22,1 & 18,4 & 10,2 & 0,3605 & 17,5 & 4,8 & 9,9 & 2,6 & 0,3674 & $-1,9$ \\
23,2 & 18,1 & 10,0 & 0,3701 & 18,3 & $-1,2$ & 10,2 & $-1,6$ & 0,3660 & 1,1 \\
24,7 & 19,8 & 12,3 & 0,4679 & 19,4 & 2,0 & 11,1 & 9,9 & 0,4939 & $-5,6$ \\
26,9 & 19,0 & 10,8 & 0,4972 & 21,0 & $-10,5$ & 11,5 & $-7,1$ & 0,5166 & $-3,9$ \\
27,5 & 23,1 & 12,4 & 0,6749 & 21,4 & 7,3 & 12,9 & $-4,2$ & 0,7143 & $-5,8$ \\
29,0 & 20,9 & 11,9 & 0,6340 & 22,5 & $-7,6$ & 12,8 & $-7,6$ & 0,6604 & $-4,2$ \\
30,1 & 21,2 & 13,7 & 0,7651 & 23,3 & $-9,8$ & 13,3 & 3,3 & 0,7854 & $-2,7$ \\
31,7 & 24,7 & 12,8 & 0,8861 & 24,4 & 1,2 & 14,9 & $-16,3$ & 0,9326 & $-5,2$ \\
32,9 & 26,0 & 13,5 & 1,0198 & 25,3 & 2,9 & 15,7 & $-16,2$ & 1,0574 & $-3,7$ \\
35,0 & 24,1 & 18,3 & 1,2264 & 26,7 & $-10,9$ & 16,1 & 12,0 & 1,2071 & 1,6 \\
36,6 & 25,7 & 19,9 & 1,4296 & 27,9 & $-8,4$ & 17,2 & 13,5 & 1,4076 & 1,5 \\
43,9 & 35,0 & 21,7 & 2,5889 & 32,9 & 5,9 & 23,5 & $-8,1$ & 2,7580 & $-6,5$ \\
\hline
\end{tabular}

Variáveis definidas anteriormente.

TABELA 7: Valores de Qui-quadrado calculado e tabelado com $(\mathrm{p} \leq 0,01)$.

TABLE 7: Values de Chi-square calculated and tabulated with $(\mathrm{p} \leq 0.01)$.

\begin{tabular}{lccc}
\hline & $\mathrm{h}(\mathrm{m})$ & $\mathrm{d}_{0,7 \mathrm{~h}}(\mathrm{~cm})$ & Volume $\left(\mathrm{m}^{3}\right)$ \\
\hline$\chi^{2}{ }_{\text {calculado }}$ & $2,60^{\mathrm{ns}}$ & $1,9^{\mathrm{ns}}$ & $0,0216^{\mathrm{ns}}$ \\
$\chi^{2}$ tabelado & 33,41 & 33,41 & 33,41 \\
\hline
\end{tabular}

Em que: ${ }^{\text {ns }}=$ não significativo $\operatorname{com}(\mathrm{p} \leq 0,01)$

\section{Validações}

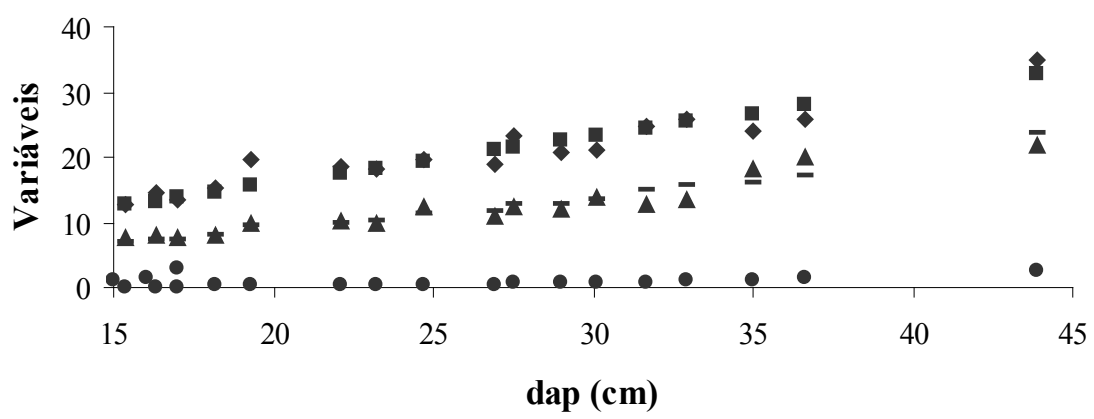

- Altura observada - Altura estimada $\Delta$ d0,7h observado

- d0,7h estimado $\quad$ Volume observado • Volume estimado

FIGURA 3: Valores observados e estimados da altura total, $\mathrm{d}_{0,7 \mathrm{~h}}$ e volume usado para validação das equações.

FIGURE 3: Observed and estimated values of the total height, $\mathrm{d}_{0.7 \mathrm{~h}}$ and volume used to validate the equations. 


\section{CONCLUSÕES}

De acordo com as condições do estudo pode-se concluir que:

A estratificação dos dados traz ganho na acurácia no ajuste da função de forma;

O polinômio do quinto grau ajustado com dados estratificados em classes de quociente de forma a $70 \%$ da altura aumenta a eficiência nas estimativas dos volumes da árvore e dos sortimentos.

A classificação dos sortimentos em função do diâmetro com altura variável segundo a relação hipsométrica mostra valores compatíveis com as dimensões das árvores, sendo esta uma ferramenta útil no planejamento da produção.

\section{REFERENCIAS BIBLIOGRÁFICAS}

ARCE, J. E. et al. Geração de padrões ótimos de corte através de algoritmos de traçamento aplicados a fustes individuais. Revista Árvore, v. 28, n. 2, p. 207-217, 2004.

ASSIS, A. L. de. et al. Comparação de modelos polinomiais segmentados e não-segmentados na estimativa de diâmetros e volumes ao longo do fuste de Pinus taeda. Cerne. v. 7, n. 1, p. 20-40, 2001.

CHICHORRO, J. F. et al. Equações de volume e de taper para quantificar multiprodutos da madeira em floresta atlântica. Revista Árvore, v. 27, n. 6, p. 799-809, 2000.
EMBRAPA. Centro Nacional de Pesquisa de Solos. Sistema brasileiro de classificação de solos. Brasília: Embrapa - SPI; Rio de Janeiro: Embrapa Solos, 2006. 306 p.

FINGER, C.A.G. Fundamentos de Biometria Florestal. 1.ed., UFSM, Santa Maria: CEPEF, 1992, $269 \mathrm{p}$.

SCHNEIDER, P. R. et al. Forma de tronco e sortimentos de madeira de Eucalyptus grandis para o estado do Rio Grande do Sul. Ciência Florestal, Santa Maria, v. 6, n. 1, p. 79-88, 1996

SCHNEIDER, P. R. et al. Estruturação da produção para o sistema de manejo de Pinus taeda L. Santa Maria, Centro de Pesquisas Florestais/FATEC, 2005.119 p. (Relatório Técnico). SOUZA, C. A. M. et al. Avaliação de modelos de afilamento segmentados na estimação da altura e volume comercial de fustes de Eucalyptus sp. Revista Árvore, Viçosa, v. 32, n. 3, p. 453-463, 2008 a.

SOUZA, C. A. M. et al. Avaliação de modelos de afilamento não-segmentados na estimação da altura e volume comercial de Eucalyptus sp. Ciência Florestal, Santa Maria, v. 18, n. 3, 2008 b.

SOUZA, C. A. M. Modelos de afilamento para Pinus taeda $\mathrm{L}$. ajustados segundo a forma do fuste e métodos de estratificação. 2009. 120 f. Tese (Doutorado em Engenharia Florestal) Universidade Federal de Santa Maria, Santa Maria, 2009. 